\title{
A robust automated method to analyze rodent motion during fear conditioning
}

\author{
Charles D. Kopec ${ }^{\text {a,1 }}$, Helmut W.H.G. Kessels ${ }^{\text {a, }}$, David E.A. Bush ${ }^{\text {b }}$, Christopher K. Cain ${ }^{\text {b }}$, \\ Joseph E. LeDoux ${ }^{\mathrm{b}}$, Roberto Malinow ${ }^{\mathrm{a}, *}$ \\ ${ }^{a}$ Cold Spring Harbor Laboratory, Jones Building, 1 Bungtown Road, Cold Spring Harbor, NY 11724, USA \\ ${ }^{\mathrm{b}}$ W.M. Keck Foundation Laboratory of Neurobiology, Center for Neural Science, New York University, 4 Washington Place, Room 809 , \\ New York, NY 10003, USA
}

Received 18 May 2006; received in revised form 7 July 2006; accepted 17 July 2006

\begin{abstract}
A central question in the study of LTP has been to determine what role it plays in memory formation and storage. One valuable form of learning for addressing this issue is associative fear conditioning. In this paradigm an animal learns to associate a tone and shock, such that subsequent presentation of a tone evokes a fear response (freezing behavior). Recent studies indicate that overlapping cellular processes underlie fear conditioning and LTP. The fear response has generally been scored manually which is both labor-intensive and subject to potential artifacts such as inconsistent or biased results. Here we describe a simple automated method that provides unbiased and rapid analysis of animal motion. We show that measured motion, in units termed significant motion pixels (SMPs), is both linear and robust over a wide range of animal speeds and detection thresholds and scores freezing in a quantitatively similar manner to trained human observers. By comparing the frequency distribution of motion during baseline periods and to the response to fox urine (which causes unconditioned fear), we suggest that freezing and non-freezing are distinct behaviors. Finally, we show how this algorithm can be applied to a fear conditioning paradigm yielding information on long and short-term associative memory as well as habituation. This automated analysis of fear conditioning will permit a more rapid and accurate assessment of the role of LTP in memory.
\end{abstract}

(C) 2006 Elsevier Ltd. All rights reserved.

Keywords: Long-term potentiation; Behavior; Fear conditioning; Automated; Motion; Memory

\section{Introduction}

Knowledge about long-term potentiation (LTP) has advanced tremendously over the last few decades. This increased understanding has occurred at the molecular, cellular and behavioral levels of analyses. Perhaps one of the most important issues raised by the initial discovery of LTP was its relation to learning and memory (Morris, 2003). This relation has been examined using a number of different experimental paradigms (Martin et al., 2000). One behavioral paradigm, associative

\footnotetext{
* Corresponding author. Tel.: +1 516367 8416; fax: +1 5163678372 .

E-mail address: malinow@cshl.edu (R. Malinow).

${ }^{1}$ These authors contributed equally to this work.
}

fear conditioning (Fanselow and Poulos, 2005; LeDoux, 2000; Maren, 2005; Rodrigues et al., 2004), has been used with considerable success as it affords a number of advantages. In this form of learning, an animal is exposed to an initially neutral conditioned stimulus (CS; generally a tone) temporally associated with an aversive unconditioned stimulus (US; usually an electrical shock). Subsequent presentations of the CS elicit an array of defensive responses, collectively referred to as conditioned fear. Behavioral freezing, defined as the absence of all non-respiratory movements, is perhaps the most widely studied conditioned fear response (Blanchard and Blanchard, 1969; Fanselow, 1980).

The advantages of this behavior, specifically in addressing the question regarding its relationship to LTP, are numerous. First, the anatomical circuitry underlying the acquisition and 
expression of this behavior is fairly well established (Blair et al., 2001; LeDoux, 2000; Maren, 2001). Thus, one can predict which synaptic contacts should undergo LTP during learning (Bauer et al., 2002; Lamprecht et al., 2006; Rumpel et al., 2005; Schafe et al., 2000, 2005) (for review see (Maren and Quirk, 2004; Rodrigues et al., 2004)). Second, the conditioning is quite brief; typically one to five tone-shock pairings are used, which mimics to some extent the LTP inducing protocols. Third, the memory is long lasting (weeks to months), as with LTP. And last, the behavioral output, freezing, is quite robust.

One drawback of this paradigm is that the behavioral output has typically been measured by human observers. This often requires multiple independent observations and is susceptible to potential bias. Furthermore, other potentially important behavioral measures, such as motion, are generally not scored. Although significant progress in automation of the analysis has been described (Contarino et al., 2002; Fitch et al., 2002; Marchand et al., 2003; Takahashi, 2004; Anagnostaras et al., 2000), these algorithms have some drawbacks. For instance, several require sophisticated hardware that measure animal movement indirectly (e.g. force-transduction or photobeam interruption). Furthermore others have poor time resolution, produce non-linear results, or only score freezing and not motion. Here we describe a reliable, low-cost method that uses digital cameras and standard speed computers to measure animal motion. The algorithm output displays a linear relation to the motion of an animal. Freezing can be calculated by setting a threshold for motion. However, the percent freezing is quite insensitive to the threshold suggesting that freezing and nonfreezing are distinct behaviors. This is further supported by comparing the behavior during unconditioned freezing and baseline behavior.

\section{Methods}

\subsection{Animals}

Male C57/B16*129 hybrid mice, 10 weeks of age, and male SpragueDawley rats (250-350 g) were housed on a 12-h light/dark cycle with ad libitum access to water and food. Procedures were performed in strict compliance with the animal use and care guidelines of Cold Spring Harbor Laboratory and New York University.

\subsection{Behavioral training}

The mice were handled and habituated to the conditioning chamber and testing chamber during $5 \mathrm{~min}$ for 5 consecutive days. Conditioning was performed in a Mouse Test Cage $(18 \mathrm{~cm} \times 18 \mathrm{~cm} \times 30 \mathrm{~cm})$ with an electrifiable floor within a larger sound attenuated cabinet (Coulbourn instruments, Allentown, PA). During conditioning the chamber was illuminated and the behavior was captured with an infrared PC-6EX2 CCD-camera (Supercircuits, Liberty Hill, TX) at $5 \mathrm{~Hz}$ and stored on a personal computer. Delivery of the tone and shock were controlled by Freezeframe ${ }^{\mathrm{TM}}$ software (Actimetrix, Wilmette, IL). $2.8 \mathrm{kHz}$ pure tones at $80 \mathrm{~dB}$ were delivered for $30 \mathrm{~s} \mathrm{co-terminating} \mathrm{with} \mathrm{a} 2 \mathrm{~s}$ $1.2 \mathrm{~mA}$ shock twice on a $1.5 \mathrm{~min}$ interval during a $5 \mathrm{~min}$ period. The memory retention test, $3 \mathrm{~h}$ after conditioning, was performed in darkness in a different shaped plastic container $(13 \mathrm{~cm} \times 13 \mathrm{~cm} \times 24 \mathrm{~cm})$ and behavior was recorded during $5 \mathrm{~min}$ in which the 30 -s tone was presented twice on a 2 min interval To measure freezing behavior during unconditioned fear, mice were first exposed to air-closed test cage $(18 \mathrm{~cm} \times 18 \mathrm{~cm} \times 30 \mathrm{~cm})$ for 2 min and subsequently exposed to the same cage for $3 \mathrm{~min}$ in the presence of fox urine (Green Sense, Garland TX). The fox urine was freshly applied $(5 \mathrm{ml})$ to a paper towel after every 2 mice tested, covering the cage floor below the raised floor on which the mice walked. Numerous small holes were drilled throughout the raised floor to allow odors to permeate up.

\subsection{Algorithm}

All analysis is performed using two custom written MatLab based programs. Videos are first converted to AVI format and fed into the first function "mousemove" which calculates the amount of motion between successive frames. The second function "mousefreeze" analyzes the output from "mousemove" and determines when the mouse is freezing verses moving based on parameters entered by the user (see below).

In "mousemove" pairs of successive frames are passed iteratively through the following algorithm. First the absolute value of the difference image is calculated. This image is divided into 16 regions (the algorithm will divide the image into between 16 and 32 regions depending on what the height and width are evenly divisible by). The mean and standard deviation are calculated for each region independently. The region with the lowest mean is designated the background region (the background region is determined independently for each pair of successive frames and therefore may be different from frame to frame). A significance threshold is set to 10 standard deviations above and below the mean. The difference image (raw difference not the absolute value difference image) is then smoothed with a Gaussian filter (sigma 0.5 pixels, size $3 \times 3$ pixels) to reduce noise and the threshold is applied to it. Any pixels within the threshold (less than 10 standard deviations from the mean) are designated non-motion pixels. Those more than 10 standard deviations from the mean are designated Significant Motion Pixels or SMPs. The number of SMPs is then counted. This process is repeated for each pair of successive frames.

The output from "mousemove" is then fed into "mousefreeze" along with the following parameters: threshold and bout length. The threshold is the SMP value below which the user considers the mouse is freezing and above which the mouse is moving. This value is usually determined empirically by watching a given mouse, observing when it begins freezing, and determining the SMP value for those frames. This value is then applied to all mice for a given experiment. The bout length defines the minimum time the mouse must stop moving to be considered a freezing bout. Here we consistently use $1 \mathrm{~s}$. Therefore, if the mouse stops moving for a fraction of a second it will not be scored as freezing.

\subsection{Availability}

The software is freely available as an open source code along with a user manual at the following URL http://malinowlab.cshl.edu/downloads/.

\section{Results}

We began by asking whether the algorithm could score the motion of a mouse in a qualitative manner. Fig. 1a shows the motion of a mouse in SMPs plotted as a function of time (see Section 2). Two successive frames from the video where the algorithm calculates a high (Fig. 1b1) or low (Fig. 1b2) SMP value are shown, indicating that SMP value does qualitatively correlate with absolution motion.

Next we asked if SMP value was a linear measure of motion. To accomplish this we designed a fake mouse consisting of a mouse drawn on a $\sim 6$ inch circular piece of filter paper mounted to a motor. By varying the voltage across the motor we could vary the speed which was calculated manually by counting how many revolutions the mouse made in $30 \mathrm{~s}$. In Fig. 1d the SMP value is plotted as a function of the rotation 
a

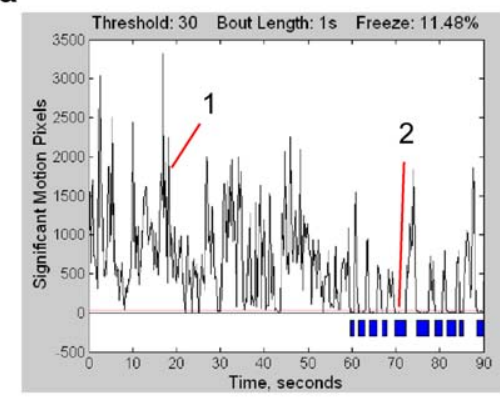

C

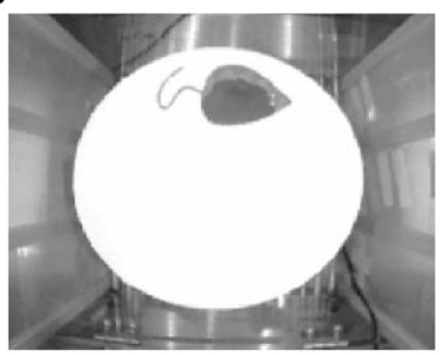

e

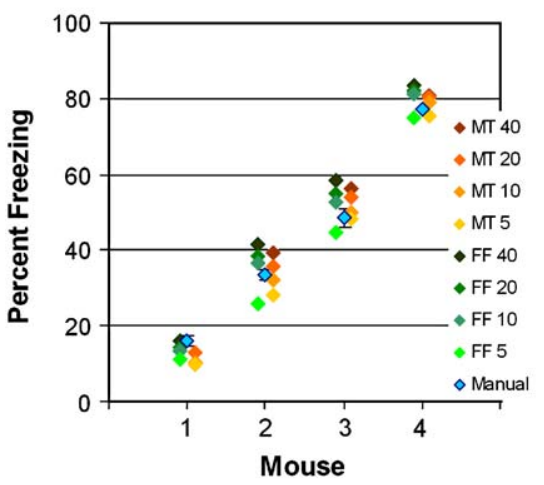

b
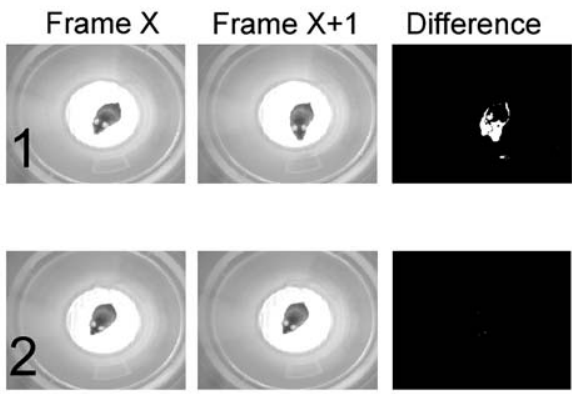

d

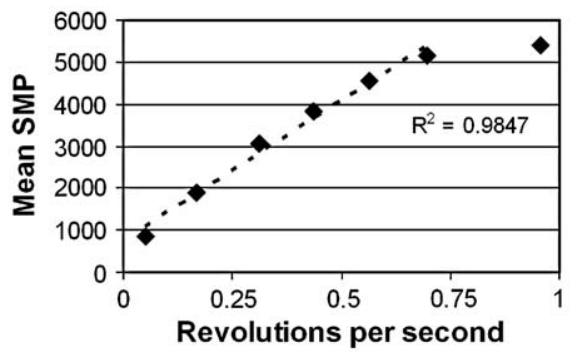

f

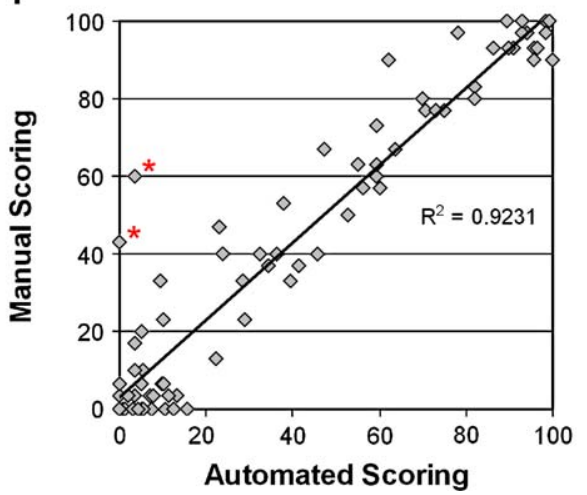

Fig. 1. The algorithm output is a linear measure of motion that scores freezing behavior in a robust manner similar to trained human observers. (a, b) Example output from a mouse in a test chamber that had been previously conditioned to associate a tone with a foot shock. First $90 \mathrm{~s}$ shown from a $300 \mathrm{~s}$ test, tone played from 60 to 90 s. (a) Output from the mousemove function, Significant Motion Pixels, SMPs, plotted as a function of time. Here a threshold of 30 SMPs and a minimum bout length of $1 \mathrm{~s}$ were used to define freezing. Blue bars designate freezing bouts. (b) Two pairs of consecutive frames from the times shown in a. The difference images are thresholded so that white pixels are SMPs and black pixels are non-motion pixels. (c, d) Fake mouse used to test if SMPs are a linear measure of motion. Voltage was increased in $30 \mathrm{~s}$ steps to the motor to cause the fake mouse to spin faster. (d) Mean SMP value from each $30 \mathrm{~s}$ step plotted verses the actual rotation velocity in revolutions per second. The linear best fit and $R^{2}$ exclude the final data point where linearity breaks down. (e) Four two minute videos of mice that showed varying levels of freezing in a context memory test where selected (arranged in linear order of increased freezing). For manual freezing the order of the mice was randomized and subjects used a stopwatch, $n=10$, error bars SEM. Freezing scored by the algorithm (MT) and the commercially available software Freeze Frame (FF) using 4 different motion thresholds $(5,10,20,40)$ is also plotted. (f) Automated verses manual scoring of freezing for 16 rats trained to associate a tone with a foot shock. Each rat received a 2 min baseline followed by $530 \mathrm{~s}$ tone presentations, freezing for each period was scored separately. Two outliers where the algorithm detected motion but where manually scored as freezing are marked by asterisks and discussed in the text. All data points including outliers were used to calculate the best fit and $R^{2}$.

velocity. The first six data points show a strong linear correlation which breaks down at the highest speed. This was expected since at a high enough velocity the mouse will not overlap with itself in consecutive frames. The SMP value will therefore be twice the area of the mouse in pixels (see Section 2). Faster speeds will yield no further increase in SMP value. This problem was never encountered during tests involving real mice but could easily be circumvented by increasing the video capture rate.
In order to score freezing, the algorithm requires the user to enter an arbitrary threshold in SMPs (below which the animal is considered freezing and above which the animal is moving). We first wanted to know how sensitive the scored freezing is to this threshold. In Fig. 1e we score freezing using four different thresholds of four different mice each showing a different amount of freezing. To achieve an absolute measure of freezing we asked 10 people to manually score freezing for each mouse and averaged their results. Qualitatively we found 
that even over an 8-fold range of thresholds the freezing scored by the algorithm was very similar to that scored manually indicating that the algorithm is very robust. Furthermore, it suggests (further explored below) that freezing behavior is distinct from slow movement (e.g. if there were a continuum of movement from fast to slow to no movement, then percent freezing would be expected to be quite sensitive to the chosen threshold). We also see that the threshold can be tuned to always score freezing to a similar degree as the average human observer. Additionally, we compared our algorithm to the commercially available software Freeze Frame, which only scores freezing and not motion. We found that over the same 8-fold range of thresholds (Fig. 1e) Freeze Frame scored freezing with a $62 \%$ larger range than our algorithm, indicating that our algorithm is less sensitive to choice of threshold. Second, we wanted to know how accurately the algorithm could score freezing compared to trained human observers. Here the threshold was determined qualitatively by following the SMP value during a single video. In Fig. 1f we plot the manually scored freezing versus that determined by the algorithm (the same threshold is used for all data points). Aside from two data points (marked by asterisks), both manually and automated scored freezing agree very well (outliers were included when calculating best fit and $R^{2}$ ). In the videos corresponding to these two points the rats took on a freezing posture but very slowly swayed their heads back and forth, which is considered as an expression of fear. This was manually scored as freezing while the program detected the motion and determined it to be significantly above the freezing threshold. The combined data indicates that SMPs are a linear measure of motion and the algorithm can score freezing for both rats and mice in a robust manner similar to trained human observers.

We next applied the algorithm on a mouse auditory fear conditioning paradigm. Mice were first habituated in both the conditioning and test cage for 5 consecutive days. The mean motion of the mice during the first three days of habituation in the shock cage is visualized in Fig. 2a. These data show that the amount of motion gradually decreases during habituation, suggesting that the time spent for exploring the cage decreases through learning. The decreased activity during habituation is associated with a rather small change in the mean percentage of scored freezing; during the habituation period of day 1 , day 2 and day 3 , freezing was scored as $1.3 \%, 2.1 \%$, and $6.9 \%$ respectively.

Next, the mice were conditioned to associate a $30 \mathrm{~s}$ tone with a foot shock. Plotting the mean SMP value per second during this conditioning protocol (Fig. 2b), shows: a) no change in mouse motion upon presentation of the first tone; b) the startle response to the foot shock; c) post-shock freezing responses to the foot shock; and d) the freezing response to the second tone played. The mean freezing responses during each 30 -s interval of the conditioning procedure are shown in Fig. 2c. The capacity to learn the association between tone and foot shock was tested by playing the tone in a different environment $3 \mathrm{~h}$ after conditioning. The fear responses to the tones are apparent by both a decrease in motion (Fig. 2d) and an increase in freezing behavior (Fig. 2e) specifically during presentation of the tone.
To address the question whether freezing behavior as a fear response is a gradual decrease in average motion, or represents a distinct behavior, mouse movement was measured under neutral circumstances and when unconditioned fear was induced. While mice do not shown any freezing when exposed to a new environment, in the presence of the odor of fox urine mice on average display freezing $38 \%$ of the time (Fig. $2 \mathrm{~g}$ ). Because frequencies of the different levels of motion during the expression of unconditioned fear displays a bimodal pattern (Fig. 2f), it appears that freezing as a fear response is not simply a result of decreased motion, but more likely represents a distinct defensive behavior.

\section{Discussion}

Understanding the relation between LTP and learning and memory is a central issue in the study of plasticity. With a molecular and cellular understanding of LTP we have gained a number of pharmacological and molecular tools that can be applied to the analysis of behavior. While the application of these tools to the study of fear conditioning has led to important findings, further progress in relating LTP to fear memory could be made through the use of automated measures that produce reliable, quantitative results at low cost.

Freezing behavior is the most commonly used measure of conditioned fear. It is often measured against a background of motion or other behavioral activity (Bouton and Bolles, 1979). Here we present an automated and quantitative measure of small animal motion that can be used to measure freezing. This method employs standard video cameras and computers. It can therefore be implemented at low cost by any modern laboratory.

We find that this algorithm calculates a value of motion that is linear with respect to the physically simulated motion of a small animal, such as a mouse or rat. To calculate percent freezing one sets a threshold, which need be done only once for each set up. The calculated percent freezing though is quite insensitive to the absolute threshold value. As shown in Fig. 1e, an eight-fold change in threshold produces little change in scored freezing. This indicates that experimental results will not strongly depend on the exact chosen threshold value. The simplicity and linearity of this method may be the greatest advantage over previous methods.

An important aspect of any automated method is the correspondence to trained human observers. In our case the correspondence was very good $\left(R^{2}=0.92\right)$. However, this movie clip was of an animal shortly after it was placed in a chamber. Animals left a long time in the chamber may display "resting" and the distinction between freezing and resting, if possible, may require human observation. It is important therefore that occasional movie clips be examined by a human observer to verify the accuracy of the algorithm. It may be possible in the future to incorporate into the algorithm more subtle differences in behavior. Currently though the algorithm is sensitive enough to detect the motion during grooming and does not score this as freezing.

By measuring motion rather than just freezing, we are able to capture more behavioral phenomena. For instance, during 
a

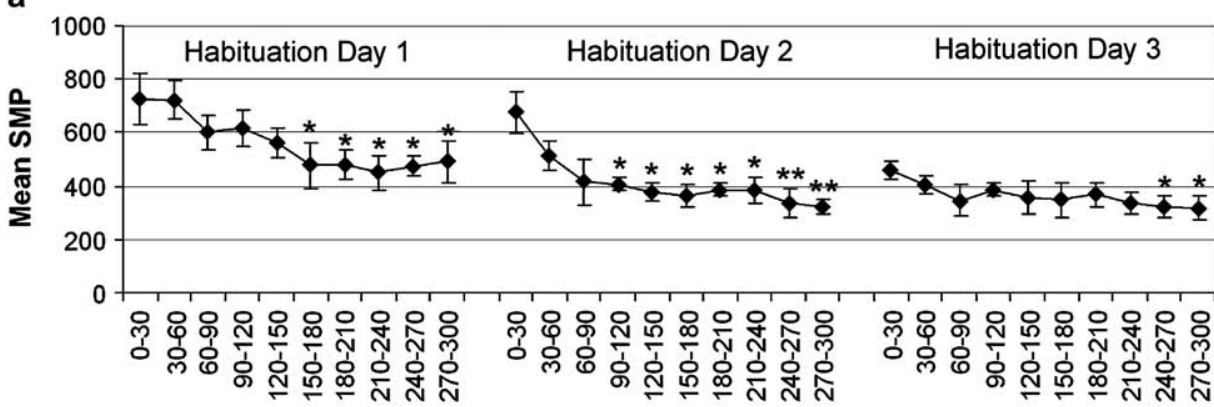

Time, seconds

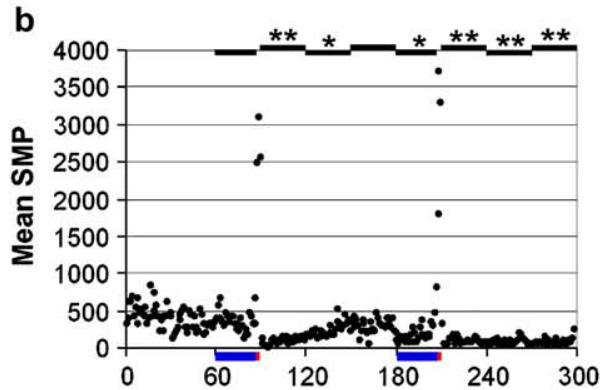

Time, seconds

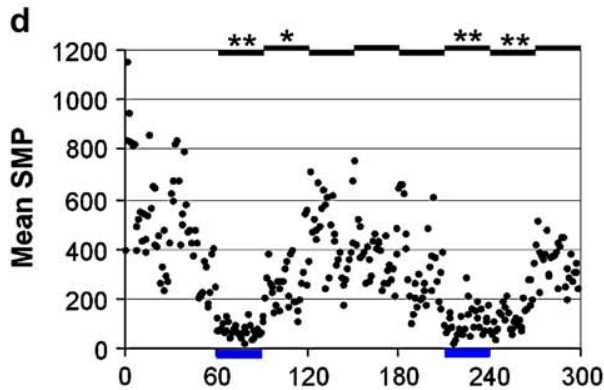

Time, seconds

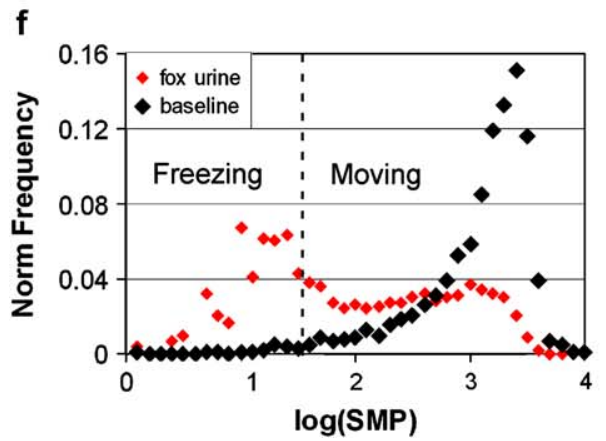

C

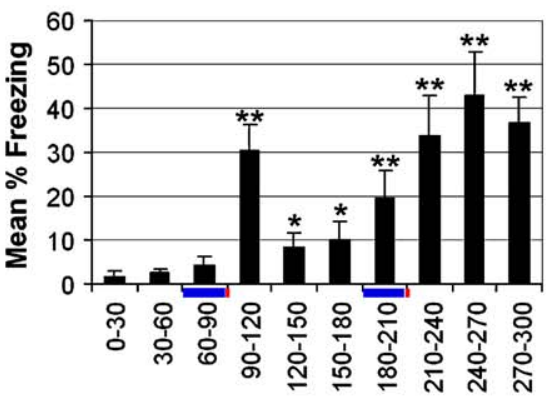

Time, seconds

e

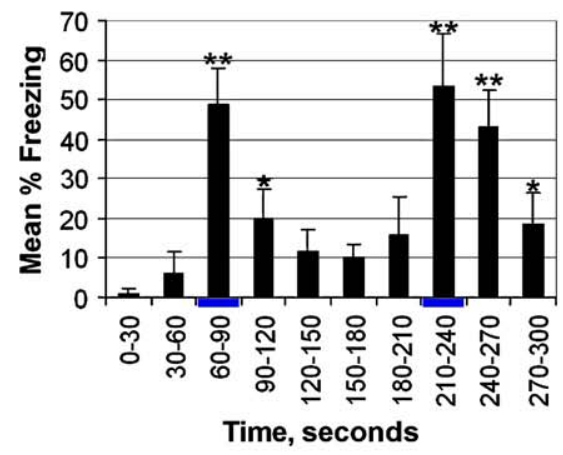

g

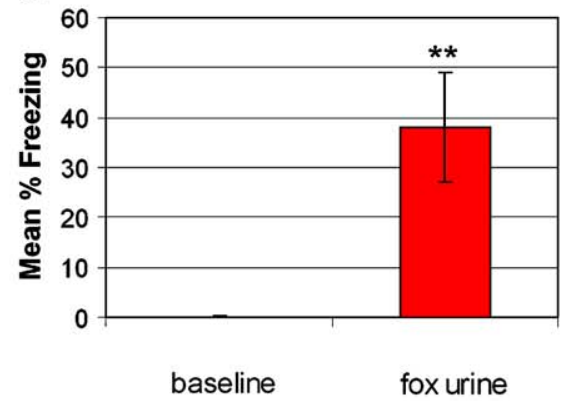

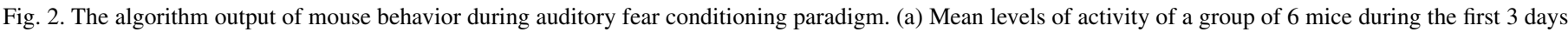

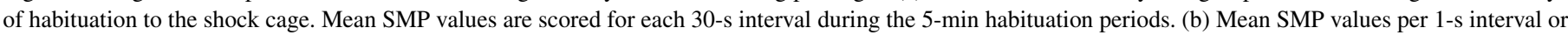

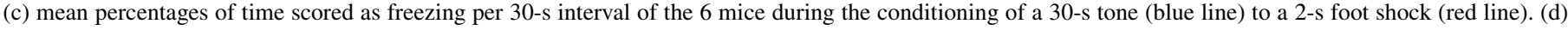

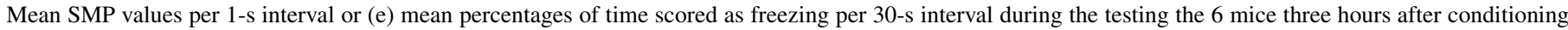

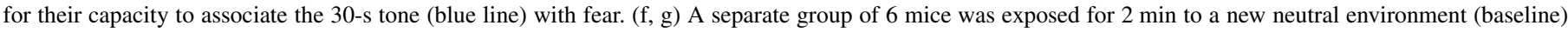

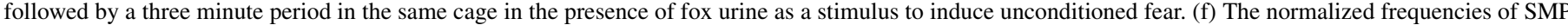

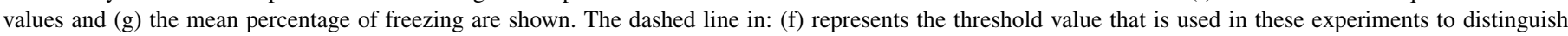

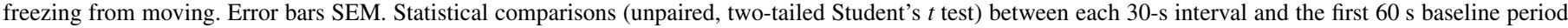
$(\mathrm{a}-\mathrm{e})$ or between presence and absence of fox urine $(\mathrm{g})$ were calculated. Values of $p<0.05(*)$ or $p<0.01(* *)$ are indicated. 
the habituation trials, there was little freezing occurring, although the motion was clearly decreasing during the trial and between trials. Another insight may be gained by measuring motion during the presentation of fox urine. As a fox is a natural predator of mice, this should produce an unconditioned freeze behavior. Indeed, the animals did display freezing. However, it is of interest that the frequency distribution of the motion approximated two Gaussian distributions; one close to zero and the other close to the movement during baseline periods. This separation of movement during exposure to fox urine suggests that freezing is a distinct behavior, and not just slow movement. Further analysis of other paradigms may shed more light on this suggestion.

\section{References}

Anagnostaras, S.G., Josselyn, S.A., Frankland, P.W., Silva, A.J., 2000. Computer-assisted behavioral assessment of Pavlovian fear conditioning in mice. Learn. Mem. 7, 58-72.

Bauer, E.P., Schafe, G.E., LeDoux, J.E., 2002. NMDA receptors and L-type voltage-gated calcium channels contribute to long-term potentiation and different components of fear memory formation in the lateral amygdala. J. Neurosci. 22, 5239-5249.

Blair, H.T., Schafe, G.E., Bauer, E.P., Rodrigues, S.M., LeDoux, J.E., 2001. Synaptic plasticity in the lateral amygdala: a cellular hypothesis of fear conditioning. Learn. Mem. 8, 229-242.

Blanchard, R.J., Blanchard, D.C., 1969. Crouching as an index of fear J. Comp. Physiol. Psychol. 67, 370-375.

Bouton, M.E., Bolles, R.C., 1979. Role of conditioned contextual stimuli in reinstatement of extinguished fear. J. Exp. Psychol. Anim. Behav. Process. $5,368-378$

Contarino, A., Baca, L., Kennelly, A., Gold, L.H., 2002. Automated assessment of conditioning parameters for context and cued fear in mice. Learn. Mem. 9, 89-96.

Fanselow, M.S., 1980. Conditioned and unconditional components of postshock freezing. Pavlov J. Biol. Sci. 15, 177-182.
Fanselow, M.S., Poulos, A.M., 2005. The neuroscience of mammalian associative learning. Annu. Rev. Psychol. 56, 207-234.

Fitch, T., Adams, B., Chaney, S., Gerlai, R., 2002. Force transducer-based movement detection in fear conditioning in mice: a comparative analysis. Hippocampus 12, 4-17.

Lamprecht, R., Margulies, D.S., Farb, C.R., Hou, M., Johnson, L.R., Ledoux, J.E., 2006. Myosin light chain kinase regulates synaptic plasticity and fear learning in the lateral amygdala. Neuroscience 139, 821-829.

LeDoux, J.E., 2000. Emotion circuits in the brain. Annu. Rev. Neurosci. 23, $155-184$.

Marchand, A.R., Luck, D., DiScala, G., 2003. Evaluation of an improved automated analysis of freezing behaviour in rats and its use in trace fear conditioning. J. Neurosci. Methods 126, 145-153.

Maren, S., 2001. Neurobiology of Pavlovian fear conditioning. Annu. Rev. Neurosci. 24, 897-931.

Maren, S., 2005. Synaptic mechanisms of associative memory in the amygdala. Neuron 47, 783-786.

Maren, S., Quirk, G.J., 2004. Neuronal signalling of fear memory. Nat. Rev. Neurosci. 5, 844-852.

Martin, S.J., Grimwood, P.D., Morris, R.G., 2000. Synaptic plasticity and memory: an evaluation of the hypothesis. Annu. Rev. Neurosci. 23, 649-711.

Morris, R.G., 2003. Long-term potentiation and memory. Philos. Trans. R. Soc. Lond. B. Biol. Sci. 358, 643-647.

Rodrigues, S.M., Schafe, G.E., LeDoux, J.E., 2004. Molecular mechanisms underlying emotional learning and memory in the lateral amygdala. Neuron $44,75-91$.

Rumpel, S., LeDoux, J., Zador, A., Malinow, R., 2005. Postsynaptic receptor trafficking underlying a form of associative learning. Science 308, 83-88.

Schafe, G.E., Atkins, C.M., Swank, M.W., Bauer, E.P., Sweatt, J.D., LeDoux, J.E., 2000. Activation of ERK/MAP kinase in the amygdala is required for memory consolidation of pavlovian fear conditioning. J. Neurosci. $20,8177-8187$.

Schafe, G.E., Bauer, E.P., Rosis, S., Farb, C.R., Rodrigues, S.M., LeDoux, J.E., 2005. Memory consolidation of Pavlovian fear conditioning requires nitric oxide signaling in the lateral amygdala. Eur. J. Neurosci. 22, 201-211.

Takahashi, H., 2004. Automated measurement of freezing time to contextual and auditory cues in fear conditioning as a simple screening method to assess learning and memory abilities in rats. J. Toxicol. Sci. 29, 53-61. 